\title{
Pheromone-Induced Accuracy of Nestmate Recognition in Carpenter Ants: Simultaneous Decrease in Type I and Type II Errors
}

\author{
Natacha Rossi, ${ }^{1, *}$, David Baracchi, ${ }^{1, \dagger}$ Martin Giurfa, ${ }^{1}$ and Patrizia d'Ettorre ${ }^{1,2}$ \\ 1. Research Center on Animal Cognition, Center for Integrative Biology, CNRS, University of Toulouse, 118 route de Narbonne, F-31062 \\ Toulouse cedex 09, France; 2. Laboratory of Experimental and Comparative Ethology, University of Paris 13, Sorbonne Paris Cité, 99 \\ avenue J.-B. Clément, Villetaneuse, France \\ Submitted April 12, 2018; Accepted September 19, 2018; Electronically published December 13, 2018 \\ Online enhancements: appendix. Dryad data: https://dx.doi.org/10.5061/dryad.14k55m8.
}

\begin{abstract}
Aвstract: The ecological and evolutionary success of social insects relies on their ability to efficiently discriminate between group members and aliens. Nestmate recognition occurs by phenotype matching, the comparison of the referent (colony) phenotype to the one of an encountered individual. Based on the level of dissimilarity between the two, the discriminator accepts or rejects the target. The tolerated degree of mismatch is predicted by the acceptance threshold model, which assumes adaptive threshold shifts depending on the costs of discrimination errors. Inherent in the model is that rejection (type I) and acceptance (type II) errors are reciprocally related: if one type decreases, the other increases. We studied whether alarm pheromones modulate the acceptance threshold. We exposed Camponotus aethiops ants to formic acid and subsequently measured aggression toward nestmates and nonnestmates. Formic acid induced both more nonnestmate rejection and more nestmate acceptance than a control treatment, thus uncovering an unexpected effect of an alarm pheromone on responses to nestmates. Nestmate discrimination accuracy was improved via a decrease in both types of errors, a result that cannot be explained by a shift in the acceptance threshold. We propose that formic acid increases the amount of information available to the ants, thus decreasing the perceived phenotypic overlap between nestmate and nonnestmate recognition cues. This mechanism for improved discrimination reveals a novel function of alarm pheromones in recognition processes and may have far-reaching implications in our understanding of the modus operandi of recognition systems in general.
\end{abstract}

Keywords: acceptance threshold model, aggressive behaviors, chemical communication, formic acid, social insects, Camponotus aethiops.

\footnotetext{
* Corresponding author; email: natacha.rossi@univ-tlse3.fr, natasha.rossi@ hotmail.fr.

${ }^{\dagger}$ These authors contributed equally to this work.

ORCIDs: Rossi, http://orcid.org/0000-0002-3631-9019; Baracchi, http://orcid .org/0000-0003-1308-0612; Giurfa, http://orcid.org/0000-0001-7173-769X; d'Ettorre, http://orcid.org/0000-0001-8712-5719.

Am. Nat. 2019. Vol. 193, pp. 267-278. (C) 2018 by The University of Chicago. 0003-0147/2019/19302-58399\$15.00. All rights reserved.

DOI: $10.1086 / 701123$
}

\section{Introduction}

Social insects have evolved highly organized societies based on division of labor and defense of colony resources. The latter relies on nestmate recognition, the ability to discriminate between nestmates and nonnestmates. This faculty favors the evolution and maintenance of sociality because it allows rejecting alien competitors (nonnestmate con- and heterospecifics) while being tolerant toward group members, which are typically related (Hamilton 1987). Apart from the rare exception of few social wasp species, where vision also plays a role in nestmate discrimination (Tibbetts 2002; Baracchi et al. 2016), the recognition of colony members in social insects is predominantly mediated by chemical cues (Lenoir et al. 1999; van Zweden and d'Ettorre 2010). Insect cuticles are covered by complex mixtures of hydrocarbons forming a waterproof layer (Blomquist and Bagnères 2010) that prevents body dehydration and plays a prominent communication role in insects living in groups (Blomquist and Bagnères 2010). Cuticular hydrocarbons (CHCs) are low volatile compounds with long carbon chain lengths (approximately $\mathrm{C}_{20}$ to $\mathrm{C}_{40}$; van Zweden and d'Ettorre 2010) that can be detected through antennal contact or at very short distances (Brandstaetter et al. 2008). Typically, CHCs vary qualitatively among species and quantitatively among colonies of the same species (vander Meer et al. 1989).

A long-standing hypothesis is that information about an individual's own CHC profile (and therefore about nestmate $\mathrm{CHC}$ profiles) is represented as a neural template in the nervous system (Lenoir et al. 1999; Leonhardt et al. 2007). During encounters with other individuals, their chemical profile (label) would be compared with the stored template in a process described as phenotype matching (Lacy and Sherman 1983). Aggressive behaviors would thus result from a mismatch between the label and the template, which is more 
likely to occur when facing a nonnestmate than a nestmate (Lacy and Sherman 1983). As CHC profiles are influenced by environmental factors, such as wax comb in honey bees, that affect both the label (d'Ettorre et al. 2006) and the template (Couvillon et al. 2007) and vary with age, reproductive status, and caste (d'Ettorre and Lenoir 2010), an adjustment of the template should occur in order to prevent rejection of nestmates with slightly different profiles. The formation and adjustment of the template are thought to require sensory adaptation or simple forms of learning (e.g., habituation; Guerrieri et al. 2009) and frequent interactions among colony members to unify the CHC label (Blomquist and Bagnères 2010).

The ability to correctly discriminate group members (typically kin) from strangers (usually nonkin) is central to the evolution and maintenance of social life (Hamilton 1987). Therefore, recognition systems have been of ongoing interest to researchers in evolutionary biology in general (e.g., Breed 2014). The study of recognition systems can be divided into three components: the expression, the perception, and the action (Reeve 1989; Sherman et al. 1997; Liebert and Starks 2004). The expression component refers to the production of the cues used for recognition; they consist of any aspect of the phenotype that predicts group membership reliably. The perception component refers to both the recognition template and the matching algorithm between the perceived cues and this template. The action component refers to the decision rules derived from recognition, the actual behavioral response. The acceptance threshold model of Reeve (1989) predicts this action component, that is, how the discriminator's action should vary in different recognition contexts. According to this model, the discriminator would have only two options when facing another individual: acceptance or rejection. The acceptance threshold is the level of phenotypetemplate dissimilarity below which the actor will accept and above which it will reject a conspecific. Because of interindividual variation and overlap between nestmate and nonnestmate recognition cues, discrimination errors are inevitable. The model predicts the acceptance threshold that optimally balances the errors of rejecting nestmates (type I) and accepting nonnestmates (type II) in different contexts. Several studies have supported the acceptance threshold model with experimental data in different social insect species. It has been shown that the acceptance threshold varies as predicted by the model with, for instance, proximity to the nest (Starks et al. 1998; Buczkowski and Silverman 2005), food availability (Downs and Ratnieks 2000), robbing intensity (Couvillon et al. 2008), stage of colony cycle (Gamboa et al. 1991), wax comb cues (d'Ettorre et al. 2006), and number of queens (Starks et al. 2010). However, there is little empirical evidence about the proximate mechanisms modulating the threshold; in particular, whether pheromones affect it and change the propensity of individuals to accept or reject nestmates and nonnestmates remains unknown.
Pheromones are substances emitted by an individual that elicit behavioral or physiological responses in individuals of the same species (Karlson and Lüscher 1959). They are used in various contexts, such as foraging, defense, and reproduction (Wyatt 2014), and are key factors in coordinating social interactions and maintaining the organization of societies, such as those of social insects (Wilson 1971; d'Ettorre and Moore 2008). Pheromones typically elicit a stereotyped response that is species specific and does not depend on experience (Karlson and Lüscher 1959; Wyatt 2014). However, recent findings indicate that the biological effects of pheromones are more complex than previously thought, as they can also act as modulators of either experiencedependent behaviors (Vergoz et al. 2007; Urlacher et al. 2010) or behaviors that are not explicitly related with the specific message they convey (Baracchi et al. 2017; Rossi et al. 2018). Therefore, pheromones appear to be key players in behavioral plasticity, an aspect that is still underexplored.

Couvillon et al. (2010) hypothesized that alarm pheromones may shift the acceptance threshold toward a less permissive one, but no significant effects of alarm pheromones on the acceptance or rejection rate of nestmates or nonnestmates by honey bee guards could be found (see "Discussion"). We thus decided to test the possible effect of formic acid (FA) on ant nestmate recognition; FA is an alarm pheromone released by most ants belonging to the Formicinae subfamily (O'Rourke 1950). It is the main component of the secretion of the venom gland (Stumper 1952), and its exposure results in increased spontaneous locomotor activity, which is part of a stereotyped alarm behavioral response (Löfqvist 1976).

We asked whether besides eliciting stereotyped responses FA modulates nestmate discrimination, in particular when the pheromone is no longer present. In a natural scenario, the situation would be that of an ant colony after an alarm situation. Does the nestmate discrimination behavior of workers change after an alert, consistent with a shift of the acceptance threshold toward a less permissive one? Does FA modulate rejection (type I) and acceptance (type II) errors? To tackle these questions, we used carpenter ants Camponotus aethiops, which use FA as alarm pheromone (Stumper 1952) and are aggressive against nonnestmates (Stroeymeyt et al. 2010). Two experiments were designed to determine whether and how FA modulates nestmate discrimination in these ants. The first experiment tested the effect of FA on ants' responsiveness to different concentrations of nonnestmate odor, which were used to mimic an odor gradient emanating from a nonnestmate depending on its distance from the receiving ant. The second experiment tested whether FA affects the level of discrimination between nestmate and nonnestmate odor cues. We conceived this experiment to test the hypothesis that an alarm pheromone not only triggers acute and stereotyped defensive responses toward potential enemies 
but also elicits a prolonged action resulting in a fine-tuned modulation of nestmate recognition behavior. In particular, this behavioral modulation would be adaptive if FA would cause an increase in effective aggressive responses toward nonnestmates and a decrease in erroneous aggressive responses toward nestmates. This hypothesis introduces a new perspective on the role played by alarm pheromones by proposing that these semiochemicals regulate defensive behavior and, additionally, modulate the attention of the defender to select appropriately the targets of its responses.

\section{Material and Methods}

Experiments were conducted in February and March 2017 at the Laboratory of Experimental and Comparative Ethology, Villetaneuse, France. We used five queen-right colonies of Camponotus aethiops collected in 2016 at Pompertuzat (Midi-Pyrénées, France; lat. 43.5, long. 1.5167) and kept in the laboratory under controlled conditions $\left(25^{\circ} \mathrm{C}\right.$; light-dark cycle of $12: 12,40 \%$ humidity), each in two Fluoncoated plastic boxes connected by a tube. One box was provided with plaster floor and covered by cardboard (nest), and the other was exposed to light and had sand on the floor (foraging area). Ants were fed twice a week with a mixture of honey and apples for carbohydrates and vitamins and pieces of crickets and flour worms for proteins; water was provided ad lib.

We conducted two experiments to determine the potential modulatory effect of FA on the perception and the associated action component of the olfactory recognition system. Experiment 1 was conducted to determine whether FA modulates responsiveness to nestmate and nonnestmate cuticular extracts. We tested different odorant concentrations to mimic an odor gradient emanating from a nonnestmate. In this case, each ant was its own control (within-subjects design), as responsiveness was measured before and after pheromone exposure. Experiment 2 was an aggressive encounter protocol, which allowed determination of whether FA modulates nestmate discrimination. We aimed at determining whether FA, besides enhancing defensive responsiveness toward nonnestmates, also affects recognition of nestmates. In this experiment, one focal ant met two target ants - a nestmate and a nonnestmate-presented simultaneously and close to each other (dual-choice situation); this design was therefore more prone to errors (both type I and type II) than when encountering a single ant and could allow observing subtle changes due to FA exposure. Target ants were previously killed by freezing so that their behavior would not influence the focal ant (C. aethiops ants are aggressive toward freshly killed nonnestmates; Stroeymeyt et al. 2010). Figure A1 (figs. A1, A2 are available online) summarizes the experimental procedure for all experiments. Data are depos- ited in the Dryad Digital Repository (Rossi et al. 2018; https://dx.doi.org/10.5061/dryad.14k55m8).

Experiment 1: Responsiveness of Ants to Recognition Cues Measured with Mandible Opening Response (MOR)

Medium-sized forager ants from two different colonies were used to study responsiveness to nestmate and nonnestmate odors (foragers are typically very aggressive in C. aethiops; Larsen et al. 2016). We used the MOR as a proxy for aggression (Guerrieri and d'Ettorre 2008; fig. A2). Each ant was cold anesthetized until immobility ( 3-4 min) and harnessed within a small plastic holder using adhesive tape placed between the head and the thorax. Ants restrained in this way can freely move only their antennae and mouthparts. Once harnessed, the ants were kept in a dark and humid cardboard box $(70 \%)$ at $25^{\circ} \pm 1{ }^{\circ} \mathrm{C}$ to recover from anesthesia and acclimatize to the harness. After the 3-h rest, ants were randomly allocated to two different groups (a control group exposed to water and an experimental group exposed to FA) and tested for their responsiveness using MOR.

In the MOR assay, one ant at a time was placed under a stereomicroscope (Leica S8 APO; magnification, $\times 10$ ). Responsiveness to nestmate and nonnestmate odors was quantified by recording the MOR to different concentrations of cuticular extracts. Cuticular extracts were obtained by washing 20 nestmate or nonnestmate ants in $10 \mathrm{~mL}$ of solvent (pentane, HPLC grade; Sigma-Aldrich) for $10 \mathrm{~min}$; concentrations were obtained by serial dilutions (see Larsen et al. 2016). The amount of nestmate odor used was equivalent to that of a single ant, while nonnestmate odor was used in six different concentrations $(0.03,0.06,0.125,0.25,0.5$, and 1 ant equivalents). An additional presentation of pure solvent (pentane) was performed as a control. The stimulus presentation was performed by placing a glass rod coated with the chemical extract next to the antennae, without touching them. The solvent evaporated prior to positioning the rod next to the antenna. At room temperature, pentane evaporates in a few seconds. The rods were let dry for $1 \mathrm{~h}$ before starting the experiment. The tips of the rods were carefully coated by adding drops of the chemical extracts using a Gilson pipette. The distance between the rod and the antennae coincided with the diameter of the rod $(1.5 \mathrm{~mm})$. The presentation lasted $1 \mathrm{~s}$. The evaluation of the response was done immediately after the rod reached the right position because usually the ants that respond do it immediately. Each ant was presented with the eight stimuli in a randomized order (pentane, nestmate extract, and six nonnestmate extracts). Each stimulus was preceded by the presentation of a clean rod in order to familiarize the ants with the visual component of this stimulus. The occurrence of MOR (yes/no) to each stimulus presentation was recorded during the presentation. 
Fifteen minutes later, ants were exposed either to FA (experimental group) or to the solvent alone (pure water, control group). FA (Sigma-Aldrich) was diluted to $12 \%$ (3 $\mu \mathrm{L}$ of pheromone plus $22 \mu \mathrm{L}$ of water, equivalent to one-third of the content of one poison gland; Stumper 1952). Control ants were exposed to $25 \mu \mathrm{L}$ of water. Ants in their plastic holders were individually confined for $15 \mathrm{~min}$ in a $50-\mathrm{mL}$ plastic flask containing a filter paper $(1 \times 5 \mathrm{~cm})$ soaked with the pheromone (or water) placed under a hood. After the exposure, ants were kept resting for an additional $30 \mathrm{~min}$ and then tested again for responsiveness using the MOR assays (fig. A1). A total of 244 ants were tested (124 ants exposed to FA and 120 ants exposed to water).

\section{Experiment 2: One-to-Two Encounters}

The arena used for aggressive encounters was a circular ring in Plexiglas $(h=5.3 \mathrm{~cm}, \varnothing=8 \mathrm{~cm})$, which delimitated a portion of the foraging area of the focal colony. It was placed in the foraging area the day before the experiment to familiarize the ants with it. The inner walls of the ring were coated with Fluon to impede the experimental ant to escape. We used three arenas, each placed in the foraging area of a colony (colonies $\mathrm{A}, \mathrm{B}$, and $\mathrm{C}$ ), and used the foragers of each colony as reciprocal nestmates and nonnestmates. On the day of the experiment, 10 ants from the focal colony and 10 ants from another colony were killed by freezing and then warmed up for $15 \mathrm{~min}$ at ambient temperature just before the experiment. One focal ant was collected from the foraging area of the focal colony and exposed either to FA (12\% in water) or to pure water. Exposure lasted $15 \mathrm{~min}$. Each focal ant was placed inside a small glass vial $(3.14 \mathrm{~mL})$ placed in the middle of a 50-mL plastic flask containing the filter paper to avoid direct contact between the ant and FA or water. Trials were therefore spaced by $15 \mathrm{~min}$ (the exposure time). We used 21 focal ants from colony A, 19 focal ants from colony B, and 29 focal ants from colony C. When focal ants from colony A were tested, nonnestmates from colony $\mathrm{C}$ were used as aggression targets; when focal ants from colony B were tested, nonnestmates from colony A were used as aggression targets; and when focal ants from colony $\mathrm{C}$ were tested, nonnestmates from colony B were used as aggression targets.

After exposure, the focal ant was placed in a Fluon-coated ring $(h=3 \mathrm{~cm}, \varnothing=2.2 \mathrm{~cm})$ inside the arena. Two target dead ants (a nestmate and a nonnestmate), presented simultaneously and interspaced at $1 \mathrm{~cm}$, were placed at one extremity of the arena, and the focal ant was released from the opposite extremity after $3 \mathrm{~min}$ of acclimatization. The respective positions of the nestmate and the nonnestmate were randomized over trials. All trials were videotaped. Target ants were marked with a small paint dot on the thorax for identification during video analysis. Trials lasted $3 \mathrm{~min}$, dur- ing which duration and occurrence of mandible opening, biting, and gaster flexing were recorded (ascending order of aggressiveness, from low to high; Stroeymeyt et al. 2010). All behaviors were exclusive, meaning that only the most aggressive behavior was recorded when two behaviors occurred at the same time. Video analysis was done blindly, as video recordings were labeled in such a way that it was not possible to know a priori the treatment to which the focal ant had been exposed. This information was obtained a posteriori, once the analysis was achieved. For video analysis, we used BORIS software (Friard and Gamba 2016). Only ants that contacted both nestmates and nonnestmates were kept in the analyses (69 in total; 35 ants exposed to FA and 34 ants exposed to water).

\section{Data Analysis}

For experiment 1, ants exhibiting inconsistent responses to nonnestmate odors (i.e., responding to a low but not to a higher concentration) were discarded (31 of the FAexposed ants [i.e., 25\%] and 34 of the water-exposed ants [i.e., $28 \%$ ]) because the lack of response to the highest concentrations may have been due to an uncontrolled motor problem and not to odor sensitivity itself. The percentage of inconsistent ants did not differ between the two treatments ( $\chi^{2}$ test, water vs. FA: $\chi^{2}=0.35$, $\mathrm{df}=1, P=.55$ ). For each ant retained in the analysis (ants exposed to FA: $n=93$; ants exposed to water: $n=86$ ), an individual MOR score was calculated as the number of nonnestmate odor stimulations eliciting MOR. MOR scores ranged from 0 to 6; that is, ants with a MOR score of 0 did not respond to any stimulation, while ants with a MOR score of 6 responded to all nonnestmate odor stimulations. Delta scores were calculated by subtracting the MOR score measured after exposure from the MOR score measured before exposure to FA (or water) and were compared using a $Z$-test, since the distribution was approximately normal and the sample size was large $(n>30)$.

Stimulus responses (MOR: 1 or 0 ) of individual ants were examined using generalized linear mixed models (GLMMs) with a binomial error structure (logit link function), using the glmer function of the R package lme4 (Bates et al. 2015). In all models colony of origin was entered as random factor, and when appropriate (i.e., repeated measures) ant individual identity was entered as a random factor nested inside colony of origin.

We first analyzed the effect of the treatment ("treatment time"; i.e., before/after exposure to either water or FA) on ants' response to nonnestmate odor concentrations ("stimulus concentration"; i.e., nonnestmate ant equivalents), using independent models for ants exposed to water and ants exposed to FA, as these groups were independent. In a second analysis, we tested the effect of the exposure to FA or 
water on ants' MOR to pentane (solvent only) and to nestmate and nonnestmate odors, both with a concentration of 1 ant equivalent ("odor stimulus"). Independent models were run for ants exposed to water and ants exposed to FA. Responses of ants to 1 ant equivalent of nestmate and nonnestmate odors were classified as either "increased aggression" or "decreased aggression" according to their variation before and after exposure to water or FA. To compare these proportions, $\chi^{2}$ tests were used. Post hoc $\chi^{2}$ tests were applied within and between treatments.

For experiment 2, we used mandible opening, biting, and gaster flexing as aggressive behaviors, and we analyzed the occurrence and duration of the sum of these three behaviors. Occurrence of aggressive behaviors was examined using GLMMs with a Poisson distribution for count data (link = 'log'; glmer function of the R package lme4), while duration of aggressive behaviors was examined using linear models (lme function of the R package nlme), with permutation tests to make sure that inferences were not biased by the nonnormal distribution of the data (PermTest function of the $\mathrm{R}$ package pgirmess; 1,000 permutations). For both "occurrence" and "duration" responses, the full models were retained, that is, with the interaction between "treatment" (FA/water) and "targeted ant" (nestmate/nonnestmate). We added "ID" as a random factor nested in "colony of origin" to account for the dependency of the data. As the interaction between "treatment" and "targeted ant" was significant for both response variables, we proceeded with models on nestmate and nonnestmate subsets separately.

In all analyses, we retained the significant model with the highest explanatory power (i.e., the lowest Akaike information criterion value). When we used post hoc tests, we applied the Bonferroni correction to correct for the familywise error rate (p.adjust function in the R package stats; $\mathrm{R}$ Development Core Team 2017). All statistical analyses were performed using $\mathrm{R}$ version 3.4.2 (R Development Core Team 2017), and the significance threshold was set at .05.

\section{Results}

\section{Experiment 1: MOR Assays}

The aggressive response of harnessed ants, expressed as MOR, increased significantly with the concentration of nonnestmate chemical extract $(0.03,0.06,0.125,0.25,0.5$, and 1 ant equivalents) in both water-exposed and FA-exposed ants: the higher the concentration, the higher the percentage of ants displaying MOR (GLMM, water-exposed group, stimulus concentration: $\chi^{2}=114.66, \mathrm{df}=5, n=86, P<$ .001; FA-exposed group, stimulus concentration: $\chi^{2}=125.33$, df $=5, n=93, P<.001$; fig. $1 A, 1 B)$. The concentration of nonnestmate odor was the only factor that had a significant effect for the water-exposed group and was consequently the only predictor retained in the model. Thus, the responses of water-exposed ants were similar before and after exposure such that this treatment did not affect the MOR to nonnestmate odors (fig. $1 B$ ). In contrast, ants were generally more likely to respond aggressively (MOR) to nonnestmate odors after being exposed to FA (GLMM, treatment time: $\chi^{2}=24.10$, df $=1, P<.001$; fig. $1 A$ ). In particular, a higher proportion of ants responded to the two lowest concentrations of nonnestmate extracts after FA exposure (GLMM, stimulus concentration $\times$ treatment time: $\chi^{2}=10.73 \mathrm{df}=$ $5, P=.057$; post hoc GLMM with Bonferroni correction: 0.03 ant equivalents, $P<.001 ; 0.06$ ant equivalents, $P=$ $.002)$.

The analysis of the MOR delta scores provides information about the variability of individual responses; it confirmed that FA affects the aggressive response of ants toward nonnestmate odors. In particular, ants exposed to the pheromone $(n=93)$ had higher delta scores than ants exposed to water $(n=86 ; Z$-test, $Z=2.80, P=.005)$, meaning that individual ants had a higher responsiveness to nonnestmate odors after FA exposure than before (fig. 1C).

We then focused on the analysis of MOR to pentane (solvent) and to 1 ant equivalent of nonnestmate and nestmate odors to represent the potential encounter with one enemy or nestmate. Pheromone exposure induced a change in the ant discrimination ability between nestmate and nonnestmate odors. In particular, FA exposure decreased MOR toward nestmates (GLMM, odor stimulus $\times$ treatment time: $\chi^{2}=17, \mathrm{df}=2, P<.001$; post hoc GLMM with Bonferroni correction: pentane, $P=.128$; nonnestmate, $P=$ .134; nestmate, $P=.032$ ). In contrast, water exposure did not affect ant responsiveness, as the treatment time factor (before/after exposure) was not retained in the model. The only predictor retained, therefore, was the odor stimulus (i.e., the chemical stimulus presented to the ants [nestmate odors, nonnestmate odors, or pentane]; GLMM, odor stimulus: $\left.\chi^{2}=77.05, \mathrm{df}=2, P<.001\right)$. This effect was also present in the group exposed to FA (GLMM, odor stimulus: $\left.\chi^{2}=56.92, \mathrm{df}=2, P<.001\right)$. In general, ants responded more to both nestmate and nonnestmate odors than to pentane as well as more to nonnestmate odors than to nestmate odors (post hoc GLMM with Bonferroni correction: in all cases, $P<.001)$.

Pheromone exposure also affected nestmate recognition accuracy. Of the 86 ants exposed to water, $6 \%$ and $14 \%$ increased their aggression level when stimulated with 1 nonnestmate equivalent and 1 nestmate equivalent, respectively. In contrast, 14\% and 19\% decreased their aggression level when stimulated with 1 nonnestmate equivalent and 1 nestmate equivalent, respectively. Of the 93 ants exposed to FA, $14 \%$ and $6 \%$ increased aggression when stimulated with 1 nonnestmate equivalent and 1 nestmate equivalent, respectively. In contrast, $5 \%$ and $24 \%$ decreased aggression when stimu- 

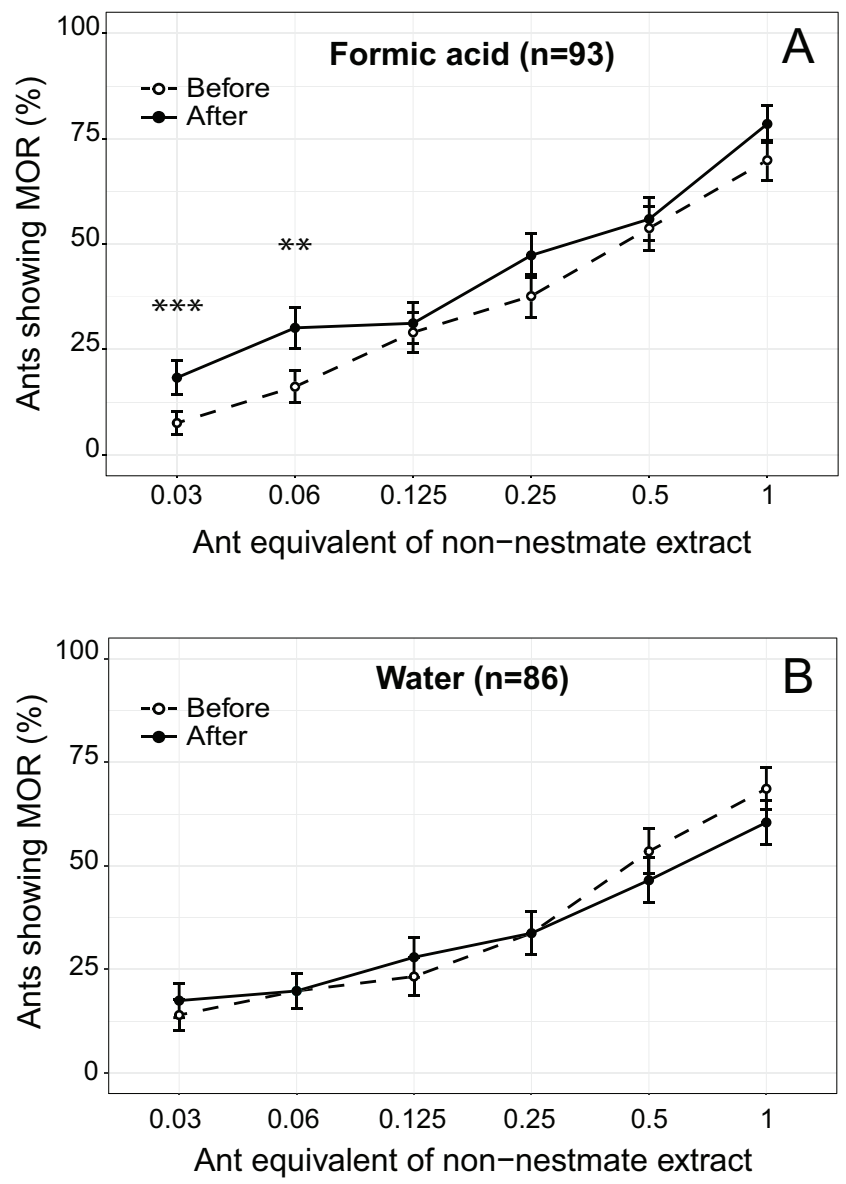

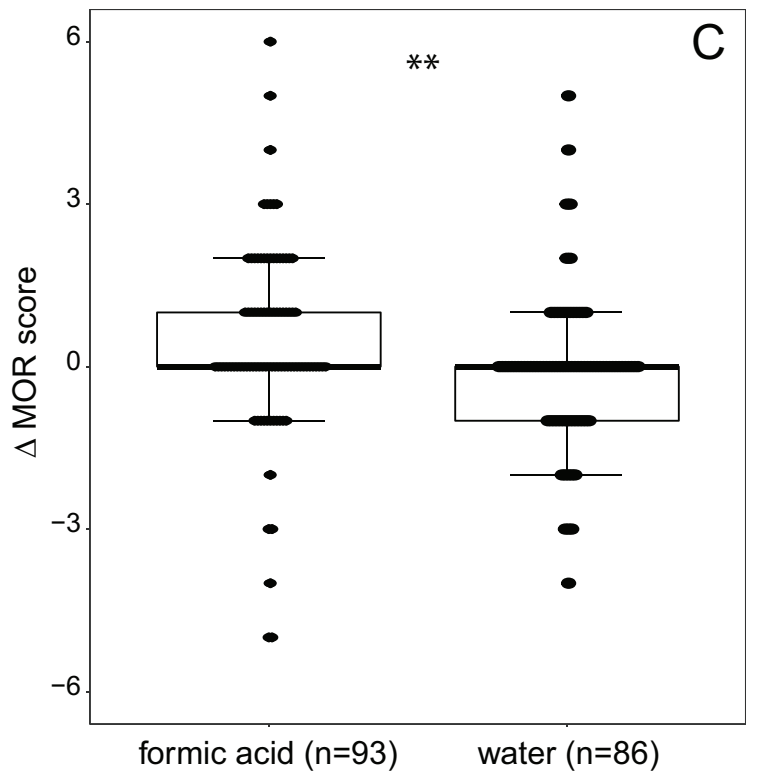

Figure 1: Pheromone exposure affects responsiveness to nonnestmate odors. $A, B$, Percentage of ants showing mandible opening response (MOR) when presented with $0.03,0.06,0.125,0.25,0.5$, and 1 ant equivalents of nonnestmate extracts. The more concentrated the extract, the more ants displayed MOR, independently of the treatment. In the formic acid (FA)-exposed group ( $A$ ), ants displayed more aggressiveness (MOR) after exposure to FA than before, in particular toward the 0.03 and 0.06 ant equivalents. No effect of water exposure $(B)$ on MOR toward the different concentrations of nonnestmate odor was detected. Means are represented with their standard errors. Post hoc generalized linear mixed model with Bonferroni correction: ${ }^{* *} P=.002,{ }^{* * *} P<.001$. C, Delta scores of ants for the six concentrations of nonnestmate odor. Scores ranged between 0 (ants responding to none of the concentrations) and 6 (ants responding to all concentrations). Delta scores were calculated by subtracting the scores measured after and before exposure to FA or water. Box plots represent median, quartiles, and 10th and 90th percentiles (lower and upper whiskers); dots represent individual ants. $Z$-test: ${ }^{* *} P=.005$.

lated with 1 nonnestmate equivalent and 1 nestmate equivalent, respectively (table 1). The number of ants that showed both an increase in aggression toward nonnestmates and a decrease in aggression toward nestmates was higher after FA exposure than after water exposure (post hoc $\chi^{2}$ test with Bonferroni correction: $\left.\chi^{2}=7.00, \mathrm{df}=1, P=.033\right)$. We then subdivided the data into changes of responses toward nestmate and nonnestmate extracts separately to see if this treatment effect was dependent on the identity of the targeted ant (fig. 2). When stimulated with the nestmate extract, the percentage of ants that stopped responding with MOR after FA exposure was higher than the percentage of ants that started responding with MOR (post hoc $\chi^{2}$ test with Bonferroni correction: $\chi^{2}=9.83$, $\left.\mathrm{df}=1, P=.021\right)$. This was not the case when stimulated with 1 nonnestmate equivalent extract (post hoc $\chi^{2}$ test with Bonferroni correction: $\chi^{2}=$ 3.82 , df $=1, P=.607$ ) or after exposure to water (post hoc $\chi^{2}$ test with Bonferroni correction, nestmate: $\chi^{2}=0.66, \mathrm{df}=$ $1, P=1$; nonnestmate: $\chi^{2}=3.35$, df $\left.=1, P=.806\right)$.

\section{Experiment 2: One-to-Two Encounters}

Ants showed more aggressive behaviors toward nonnestmates than toward nestmates (GLMM, $\chi^{2}=87.89$, $\mathrm{df}=$ $1, P<.001)$, but the magnitude of their responses was modulated by the treatment (FA or water exposure: GLMM, $\chi^{2}=6.08, \mathrm{df}=1, P=.014$; fig. $3 A$ ). In particular, the effect of FA depended on whether the targeted ant was a 
Table 1: Number of ants that increased or decreased aggression to one nestmate or nonnestmate equivalent following water or formic acid exposure

\begin{tabular}{lcccc}
\hline & & \multicolumn{2}{c}{ Change in aggression } & \\
\cline { 3 - 3 } & No change & Increased & Decreased & Total changed \\
\hline Water: & & & & \\
$\quad$ Nonnestmate & $69(80)$ & $5(6)$ & $12(14)$ & $17(20)$ \\
$\quad$ Nestmate & $58(67)$ & $12(14)$ & $16(19)$ & $28(33)$ \\
$\begin{array}{l}\text { Formic acid: } \\
\quad \text { Nonnestmate }\end{array}$ & $75(81)$ & $13(14)$ & $5(5)$ & $18(19)$ \\
$\quad$ Nestmate & $65(70)$ & $6(6)$ & $22(24)$ & $28(30)$ \\
\hline
\end{tabular}

Note: Data are no. (\%).

nestmate or a nonnestmate (GLMM, $\chi^{2}=19.68$, $\mathrm{df}=1$, $P<.001)$. Formic acid-exposed ants were less aggressive toward nestmates than water-exposed ants (GLMM, $\chi^{2}=$ $9.14, \mathrm{df}=1, P=.002)$, while they were more aggressive toward nonnestmates than water-exposed ants (GLMM, $\chi^{2}=12.73$, df $\left.=1, P<.001\right)$. Regarding the duration of aggressive behaviors, the analysis revealed very similar results (fig. 3B). Thus, compared with water exposure, FA exposure increased differentiation between nestmates and nonnestmates, triggering in each case the appropriate response (nonaggression and aggression, respectively).

\section{Discussion}

Our study aimed at investigating the role of FA as a possible modulator of nestmate discrimination in the ant Camponotus aethiops. To this end, we exposed ants to FA and de- termined its effect on the ability to discriminate nestmates and nonnestmates when the pheromone was no longer present. We assessed response modulation rather than acute responses to FA in two independent experiments. The first experiment relied on a protocol for harnessed ants, which also allowed testing for responsiveness to different concentrations of nonnestmate odor using MOR as a proxy for aggression. We found that FA increased the aggressive response of ants and in particular improved responsiveness to low concentrations of nonnestmate odor. The second experiment relied on a protocol for free-walking ants, which allowed testing ants in an experimental arena in one-to-two encounters. This arena was located within the foraging area of the colony and therefore provided a suitable scenario for potential agonistic encounters with intruders hunting for colony resources. When FA-exposed ants encountered a nestmate and a nonnestmate at the same time, they both increased aggressive behaviors to-

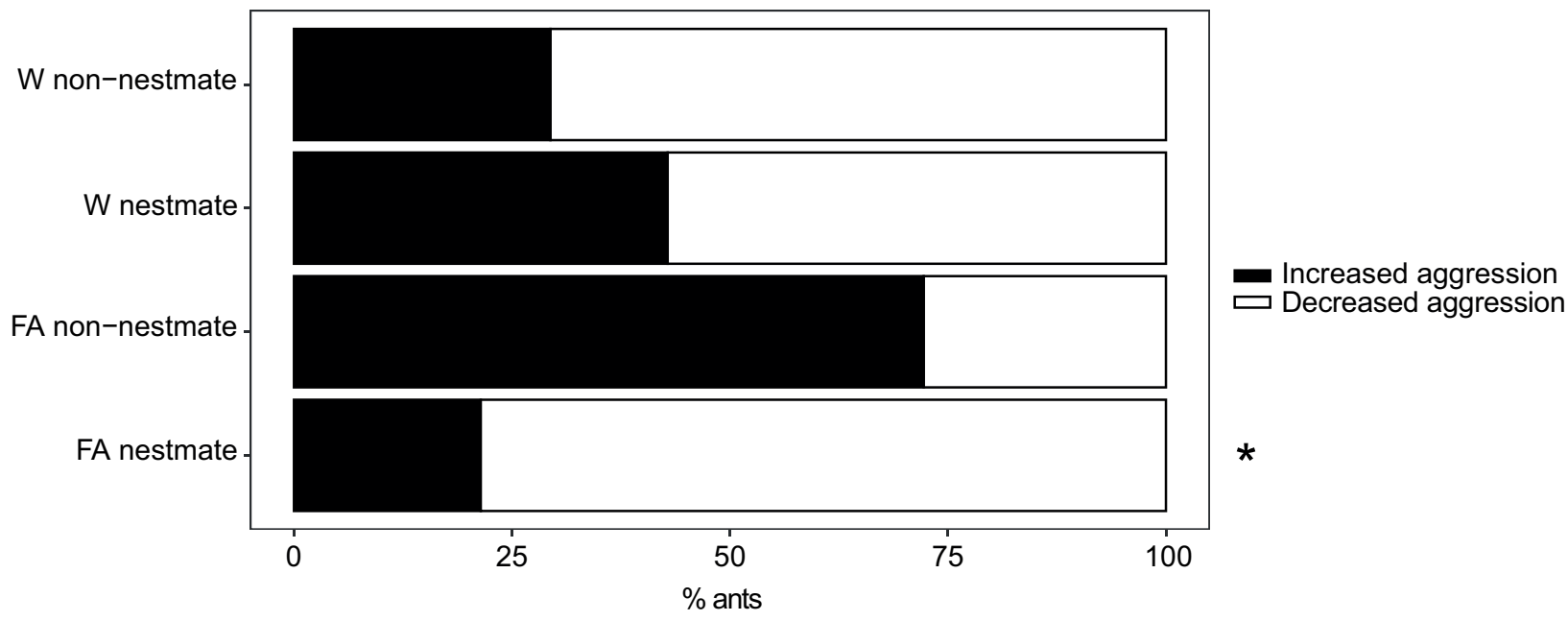

Figure 2: Percentage of ants that changed their mandible opening response (MOR) to 1 ant equivalent of nestmate and nonnestmate extracts after water (W) or formic acid (FA) exposure. Change is expressed in terms of the percentage of ants that increased or decreased MOR on presentation of nestmate/nonnestmate odor. Water exposure did not induce significant differences between the increase and the decrease in aggressive behavior to both a nonnestmate (first row) and a nestmate (second row) ant equivalent. In contrast, FA exposure tended to increase aggression toward nonnestmate odor (third row) and induced significant differences between the increase and the decrease in aggression toward the nestmate odor (fourth row). Post hoc $\chi^{2}$ test with Bonferroni correction: ${ }^{*} P=.021$. 


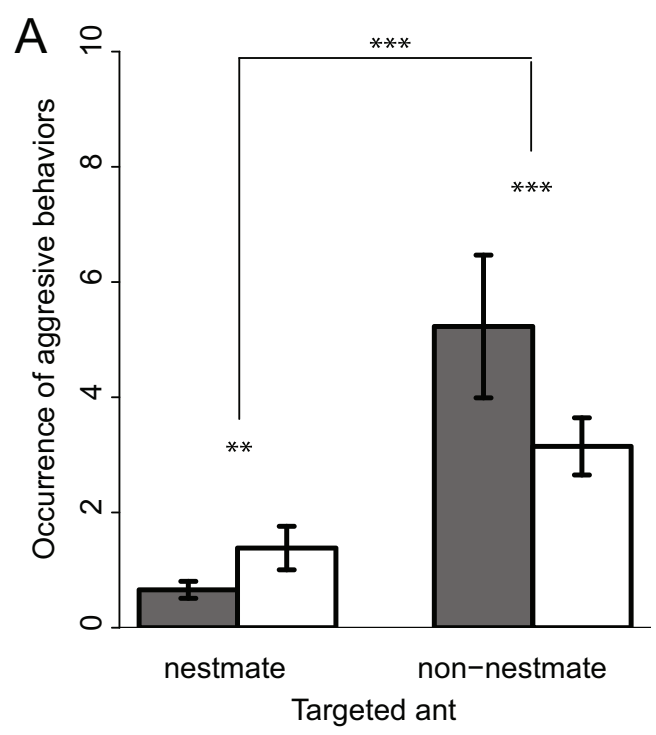

$\square \quad$ formic acid $(n=35)$
$\square \quad$ water $(n=34)$

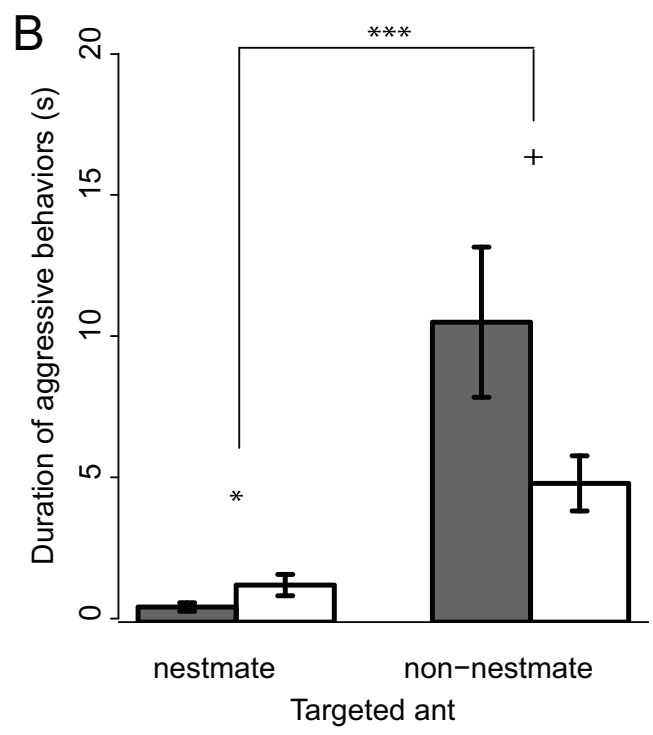

formic acid $(n=35)$

water $(n=34)$

Figure 3: Formic acid (FA) increases aggressive behaviors toward nonnestmates and decreases aggressive behaviors toward nestmates. $A$, In one-to-two encounters, FA-exposed ants displayed aggressive behaviors more frequently toward nonnestmates and less frequently toward nestmates than water-exposed ants. Generalized linear mixed model: ${ }^{* * *} P<.01,{ }^{* * *} P<.001$. $B$, Ants exposed to FA spent less time in aggressive interactions with nestmates than water-exposed ants (linear mixed model $[\mathrm{LMM}]$ with permutation test, $\chi^{2}=4.06, \mathrm{df}=1, P=.045$ ) but showed a tendency to spend more time in aggressive interactions with nonnestmates (LMM with permutation test, $\chi^{2}=3.49$, df $=1$, $P=.065)$. Means are represented with their standard errors. LMM with permutation test: ${ }^{+} P<.1,{ }^{*} P<.05,{ }^{* * * *} P<.001$.

ward nonnestmates and decreased them against nestmates, compared with water-exposed ants. This unforeseen effect of FA was also found in the first experiment, where ants decreased recognition errors toward cuticular extracts of nestmates. FA increases accuracy in the process of nestmate discrimination. We thus describe a novel function of an insect pheromone that is highly relevant in the context of colony defense, where nestmates and nonnestmates are encountered at the same time around the nest.

Alarm pheromones in social insects have been typically considered acute signals used in a defensive context, which are essential for colony survival (Blum 1985). For social species, alarm pheromones constitute a major evolutionary achievement that allows the collective resources of the colony to be rapidly recruited in response to a threat. Different strategies and modes of action are observed. In large colonies, alarm behavior is a syndrome characterized by the rapid attraction of aggressive workers to the source of pheromonal emission followed by attacks toward intruders. For small colonies, alarm pheromones also constitute an ineludible signal necessary for colonial survival, but in this case they may prompt dispersion in response to threats instead of a display of overt aggressive behaviors (Blum 1985).
Our results add a new twist to the traditional view of alarm pheromones by including a long-term action and responses to nestmates as a behavior potentially targeted by FA. In this novel framework, the function of alarm pheromones would be more comprehensive than previously thought: not only do they enhance aggressive responses toward potentially noxious stimuli and enemies but they also decrease erroneous aggressive responses toward nestmates, which should be preserved during a contest.

Testing this hypothesis in other social insects employing different alarm pheromones would reveal whether this double effect of alarm pheromones is conserved across species or is a specificity of ants using FA. A first attempt has been done in honey bees, where alarm pheromone components isopentyl acetate and 2-heptanone were delivered to the hive entrance to guard bees presented with a nestmate or a nonnestmate (Couvillon et al. 2010). In this case, no effect of alarm pheromones on the percentage of nestmate/nonnestmate acceptance was found, but the apparent discrepancy with our results could be explained by considering the different behavioral responses recorded in the two studies. While Couvillon et al. (2010) scored the behavior of guard honey bees as an absolute binary response (accept/reject) during 
pheromone exposure, we quantified a range of aggressive behaviors in one-to-two encounters (experiment 2) and the MOR variation following pheromone exposure (i.e., before vs. after exposure; experiment 1). Having an individual reference level (within-subjects design) for estimating response variation may provide a fine-tuned analysis of the effect of alarm pheromones. Alternatively, the double effect found for FA may be specific for this substance and species.

Our results show that FA affects aggressive responsiveness to nonnestmate cues when these are presented in low but not high concentrations (fig. 1A). This differential modulation is commonly found in different forms of behavioral responsiveness that are enhanced by pheromones (e.g., honeybee sting extension responses to electric shock of increasing voltages; Tedjakumala et al. 2014; Rossi et al. 2018). Higher levels of the stimulus triggering responsiveness (i.e., concentration of nonnestmate cuticular chemical extract) may bring responsiveness close to its maximum, thus rendering the observation of fine enhancements difficult. In contrast, lower levels of stimulation inducing low responsiveness facilitate the observation of modulatory enhancements.

What are the neural mechanisms underlying the observed effect of FA? Our behavioral results do not allow a direct answer to this question in the absence of neural analyses identifying the circuits underlying pheromone modulation in the ant brain. Yet we provide information here that may be useful for designing future experiments to this end. Information from olfactory receptor neurons located within cuticular structures of the antennae termed sensilla is conveyed to the antennal lobes, where inhibitory local interneurons reshape the olfactory message, thereby enhancing the contrast between glomeruli, the constitutive units of the antennal lobes. From the antennal lobes, information is passed via projection neurons to higher-order brain centers (e.g., mushroom bodies and lateral horns). FA might affect the sensitivity to recognition cues by modulating the responses to $\mathrm{CHC}$ of olfactory receptor neurons. The ant cuticular signature consists of dozens of CHCs ( 40 in $C$. aethiops; van Zweden and d'Ettorre 2010), but at very low concentrations (e.g., when the target ant is at some distance) only the most abundant $\mathrm{CHCs}$ will be detected. The modulatory effect of FA may consist in an improvement of $\mathrm{CHC}$ detection and perception and thus in an increase of the amount of information (e.g., the number of detected CHCs) available to the ant (fig. 4). In Camponotus ants, FA is processed by a set of specific glomeruli in the antennal lobes (Mizunami et al. 2010), with uniglomerular projection neurons. This specific FA pathway could mediate aggressive behaviors, triggered by CHCs (Mizunami et al. 2010). In addition, improved discrimination between nestmates and nonnestmates could be due to the ability of FA to modulate neural modulatory pathways distinct from those of olfactory circuits but affecting activity in these circuits.
Candidates for this action could be biogenic amine pathways (dopaminergic, octopaminergic, serotonergic), which are known to modulate the response of olfactory neurons at both the peripheral level (olfactory receptors; Pophof 2000 , 2002) and the central level (Sachse and Galizia 2002; Jefferis et al. 2007). Interestingly, some of these pathways, such as the dopaminergic and serotonergic pathways, have been shown to modulate attentional processes in bees and flies (Van Swinderen and Andretic 2011; Tedjakumala et al. 2014).

Even though our results do not allow identification of the precise mechanisms of action of FA, they help us understand how recognition systems operate in general. The acceptance threshold model (Reeve 1989) states that, when the cue distribution of nestmates and nonnestmates overlap, there is a risk of error. The behavior of the discriminating individual is an all-or-none response with a threshold above which all recipients are rejected. If the threshold is too restrictive, the discriminator runs the risk of erroneously rejecting desirable recipients (type I errors); if the threshold is too permissive, the risk is for erroneous acceptance of undesirable recipients (type II errors). According to this model, regardless of which direction this threshold moves, in no case is it possible to obtain both an increase in rejection frequency of nonnestmates and a decrease in rejection frequency of nestmates: if rejection errors increase, acceptance errors decrease, and vice versa. However, our results show that an alarm pheromone induces a decrease in both types of error. Hence, it seems unlikely that alarm pheromones simply shift this threshold. This apparent incompatibility between the model predictions and the observed alarm pheromone modulation of nestmate recognition in ants can be reconciled by focusing on a sentence in Reeve (1989, p. 409): "Discrimination errors are inevitable whenever phenotype matching is based on a finite set of cues" (emphasis added). We propose that alarm pheromones act on this set of cues by increasing the amount of information available to the individual performing the discrimination. Specifically, alarm pheromones would lower a perception threshold, allowing the discriminator to perceive more cues. This increase of information would be associated with an increase in the probability of detecting differences between a perceived label and the internal template of the discriminator, which would result, in the case of social insects, in a better discrimination between nestmates and nonnestmates (fig. 4A). This means a decrease in variance and possibly a shift in the dissimilarity mean values for nestmates and nonnestmates, thereby decreasing both their perceived overlap and the resulting acceptance and rejection errors (fig. 4B). Our hypothesis is supported by a model by Lehmann and Perrin (2002) showing that the distributions of similarities between a recipient and the discriminator depend on the number of recognition traits sampled: sampling a high number of traits decreases overlap and thus 
A
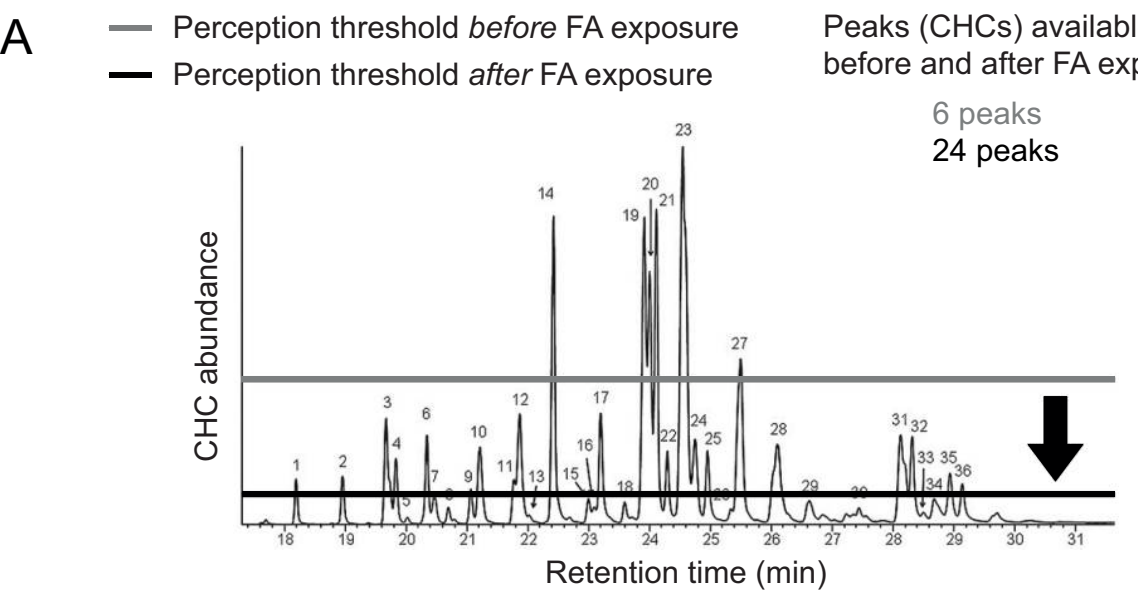

Peaks (CHCs) available

before and after FA exposure:

6 peaks

24 peaks

\section{B II Acceptance error before FA exposure WI Rejection error before FA exposure Acceptance error after FA exposure Rejection error after FA exposure}

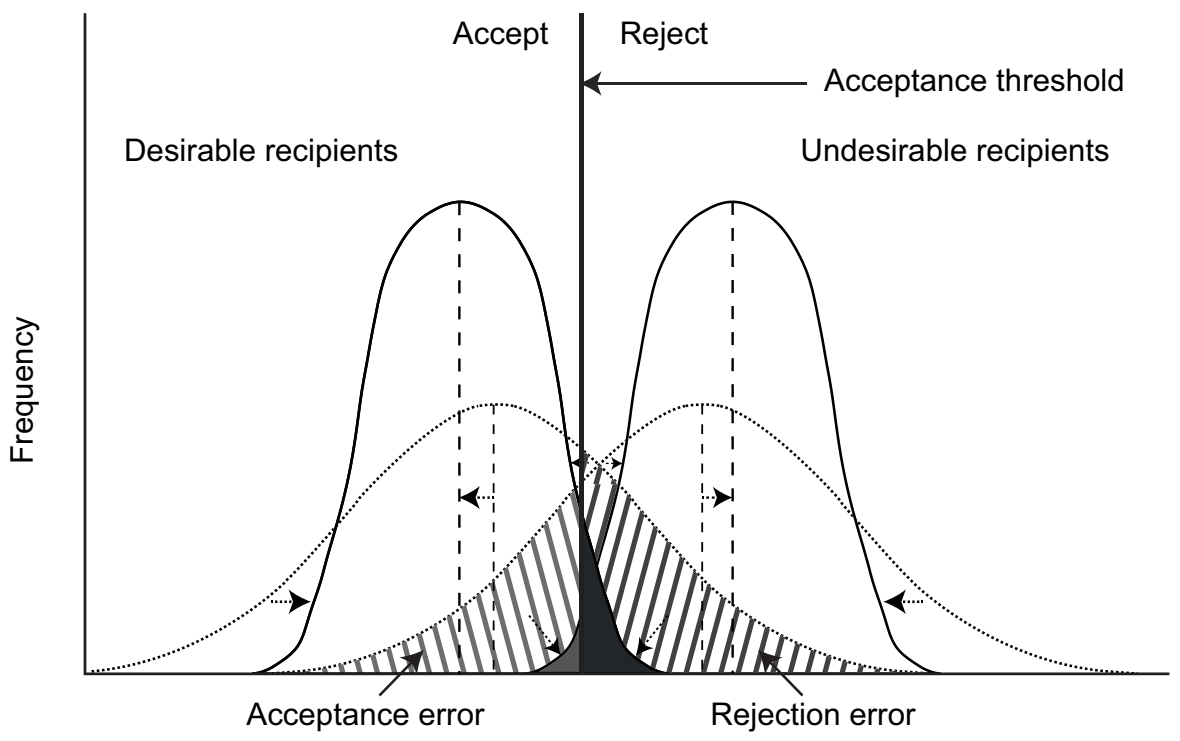

Perceived dissimilarity between recipient and template

Figure 4: Putative modulation of an olfactory perception threshold by formic acid (FA). A, Example of a cuticular profile (adapted from van Zweden et al. 2009): the $X$-axis represents the retention time of different components of the cuticular profile (cuticular hydrocarbons [CHCs] ) in a gas chromatograph, and the $Y$-axis represents their abundance. After exposure to FA, the olfactory perception threshold of an ant (horizontal line) would be lowered (black arrow), allowing detection of a higher number of components. $B$, Frequency of cues that differ between the discriminator's template and the recipient's phenotype for undesirable (nonnestmate) and desirable (nestmate) recipients (adapted from van Zweden and d'Ettorre 2010). By lowering the olfactory perception threshold, FA would allow ants to access more cues, thereby decreasing the perceived variance (dotted arrows) and accentuating the dissimilarity between nestmate and nonnestmate (dotted arrows) CHCs. This would result in the decrease of acceptance and rejection errors (dotted arrows). Vertical dashed lines represent mean values of perceived dissimilarity between recipient and template for desirable and undesirable recipients.

increases discrimination ability. According to Sherman et al. (1997), the changes in magnitudes of and balance between acceptance and rejection errors are either the result of changes in the recognition cues (production component) or of changes in the recognition template or matching algorithm (perception component). Alarm pheromone-induced changes in the pro- duction of CHCs in social insects seem highly unlikely due to the rapid action of the pheromone. Therefore, the most likely scenario is that FA acts at the perception level, as we argued above. Alarm pheromones would thus provide an adaptive mechanism to increase the amount of perceived information in the crucial defensive context, which might be less costly in 
terms of brain processing than having to apprehend all olfactory cues available at any time.

To our knowledge, few works have discussed recognition models from a perceptual perspective because accessing an animal's brain on an interindividual recognition task is difficult. Our study shows that behavior can provide clues about mechanisms that are not necessarily related to the action component. The amount of information available for decision-making is not necessarily fixed. Selection can act by changing the recognition cues used (Crozier 1979; Holman et al. 2013) or by changing the template cue matching algorithm (Sherman et al. 1997). Our study suggests that, at the perceptual level, this amount of information can be modulated by "priming," that is, exposure to one stimulus that influences a response to a subsequent stimulus. The priming effect is not restricted to pheromones and can be generally observed in all organisms that require recognition of self, kin, mates, neighbors, prey, predators, and so on to survive and reproduce. Odors may influence human behavior via the modulation of other sensory inputs (visual, auditory; Castiello et al. 2006; d'Ettorre et al. 2018; reviewed in Bueno and Frenck-Mestre 2008). If we smell orange odor, it is easier to identify an orange in a complex visual scene (e.g., among 12 other items; Seigneuric et al. 2010). Taking into account the priming effect of pheromones opens new perspectives for a deeper understanding of recognition systems and decisionmaking in general.

\section{Acknowledgments}

We thank Heiko G. Rödel for guidance with the generalized linear mixed models. Funding was received from the Agence Nationale de la Recherche (ANR-14-CE18-0003, PHEROMOD), IDEX initiative "Chaire d'Attractivité," the University of Toulouse (to P.d'E.), and the Centre National de la Recherche Scientifique (CNRS). M.G. thanks the CNRS and the University of Toulouse for their support. We report no competing interests.

\section{Literature Cited}

Baracchi, D., J.-M. Devaud, P. d'Ettorre, and M. Giurfa. 2017. Pheromones modulate reward responsiveness and non-associative learning in honey bees. Scientific Reports 7:1-31.

Baracchi, D., S. Turillazzi, and L. Chittka. 2016. Facial patterns in a tropical social wasp correlate with colony membership. Science of Nature 103:80.

Bates, D., M. Maechler, B. Bolker, and S. Walker. 2015. Fitting linear mixed-effects models using lme4. Journal of Statistical Software $67: 1-48$.

Blomquist, G. J., and A.-G. Bagnères. 2010. Insect hydrocarbons: biology, biochemistry, and chemical ecology. Cambridge University Press, Cambridge.
Blum, M. S. 1985. Alarm pheromone. Pages 193-224 in G. A. Kerkut and L. I. Gilbert, eds. Comparative insect physiology, biochemistry and pharmacology. Pergamon, Oxford.

Brandstaetter, A. S., A. Endler, and C. J. Kleineidam. 2008. Nestmate recognition in ants is possible without tactile interaction. Naturwissenschaften 95:601-608.

Breed, M. D. 2014. Kin and nestmate recognition: the influence of W. D. Hamilton on 50 years of research. Animal Behaviour 92:271279.

Buczkowski, G., and J. Silverman. 2005. Context-dependent nestmate discrimination and the effect of action thresholds on exogenous cue recognition in the Argentine ant. Animal Behaviour 69:741-749.

Bueno, S., and C. Frenck-Mestre. 2008. The activation of semantic memory: effects of prime exposure, prime-target relationship, and task demands. Memory and Cognition 36:882-898.

Castiello, U., G. M. Zucco, V. Parma, C. Ansuini, and R. Tirindelli. 2006. Cross-modal interactions between olfaction and vision when grasping. Chemical Senses 31:665-671.

Couvillon, M. J., S. N. Barton, J. A. Cohen, O. K. Fabricius, M. H. Kärcher, L. S. Cooper, M. J. Silk, H. Helanterä, and F. L. W. Ratnieks. 2010. Alarm pheromones do not mediate rapid shifts in honey bee guard acceptance threshold. Journal of Chemical Ecology 36:1306-1308.

Couvillon, M. J., J. P. Caple, S. L. Endsor, M. Kärcher, T. E. Russell, D. E. Storey, and F. L. W. Ratnieks. 2007. Nestmate recognition template of guard honeybees (Apis mellifera) is modified by wax comb transfer. Biology Letters 3:228-230.

Couvillon, M. J., E. J. H. Robinson, B. Atkinson, L. Child, K. R. Dent, and F. L. W. Ratnieks. 2008. En garde: rapid shifts in honeybee, Apis mellifera, guarding behaviour are triggered by onslaught of conspecific intruders. Animal Behaviour 76:1653-1658.

Crozier, R. H. 1979. Genetics of sociality. Pages 223-286 in H. R. Hermann, ed. Social insects. Vol. 1. Academic Press, New York.

d'Ettorre, P., S. Bueno, H. G. Rödel, H. Megherbi, A. Seigneuric, B. Schaal, and S. C. Roberts. 2018. Exposure to androstenes influences processing of emotional words. Frontiers in Ecology and Evolution 5:169.

d'Ettorre, P., and A. Lenoir. 2010. Nestmate recognition. Pages 194209 in L. Lach, C. L. Parr, and K. L. Abbott, eds. Ant ecology. Oxford University Press, New York.

d'Ettorre, P., and A. J. Moore. 2008. Chemical communication and the coordination of social interactions in insects. Pages 81-96 in P. d'Ettorre and D. P. Hughes, eds. Sociobiology of communication: an interdisciplinary perspective. Oxford University Press, New York.

d'Ettorre, P., T. Wenseleers, J. Dawson, S. Hutchinson, T. Boswell, and F. L. W. Ratnieks. 2006. Wax combs mediate nestmate recognition by guard honeybees. Animal Behaviour 71:773-779.

Downs, S. G., and F. L. W. Ratnieks. 2000. Adaptive shifts in honey bee (Apis mellifera L.) guarding behavior support predictions of the acceptance threshold model. Behavioral Ecology 11:326-333.

Friard, O., and M. Gamba. 2016. BORIS: a free, versatile open-source event-logging software for video/audio coding and live observations. Methods in Ecology and Evolution 7:1325-1330.

Gamboa, G. J., R. L. Foster, J. A. Scope, and A. M. Bitterman. 1991. Effects of stage of colony cycle, context, and intercolony distance on conspecific tolerance by paper wasps (Polistes fuscatus). Behavioral Ecology and Sociobiology 29:87-94.

Guerrieri, F. J., and P. d'Ettorre. 2008. The mandible opening response: quantifying aggression elicited by chemical cues in ants. Journal of Experimental Biology 211:1109-1113. 
Guerrieri, F. J., V. Nehring, C. G. Jørgensen, J. Nielsen, C. G. Galizia, and P. d'Ettorre. 2009. Ants recognize foes and not friends. Proceedings of the Roval Society B 276:2461-2468.

Hamilton, W. D. 1987. Discrimination nepotism: expectable, common, overlooked. Pages 417-437 in D. J. C. Fletcher and C. D. Michener, eds. Kin recognition in animals. Wiley, New York.

Holman, L., J. S. van Zweden, T. A. Linksvayer, and P. d'Ettorre. 2013. Crozier's paradox revisited: maintenance of genetic recognition systems by disassortative mating. BMC Evolutionary Biology $13: 211$.

Jefferis, G. S. X. E., C. J. Potter, A. M. Chan, E. C. Marin, T. Rohlfing, C. R. Maurer, and L. Luo. 2007. Comprehensive maps of Drosophila higher olfactory centers: spatially segregated fruit and pheromone representation. Cell 128:1187-1203.

Karlson, P., and M. Lüscher. 1959. "Pheromones": a new term for a class of biologically active substances. Nature 183:55-56.

Lacy, R. C., and P. W. Sherman. 1983. Kin recognition by phenotype matching. American Naturalist 121:489-512.

Larsen, J., V. Nehring, P. d'Ettorre, and N. Bos. 2016. Task specialization influences nestmate recognition ability in ants. Behavioral Ecology and Sociobiology 70:1433-1440.

Lehmann, L., and N. Perrin. 2002. Altruism, dispersal, and phenotypematching kin recognition. American Naturalist 159:451-468.

Lenoir, A., D. Fresneau, C. Errard, and A. Hefetz. 1999. Individuality and colonial identity in ants: the emergence of the social representation concept. Pages 219-237 in C. Detrain, J. L. Deneubourg, and J. M. Pasteels, eds. Information processing in social insects. Birkhäuser, Basel.

Leonhardt, S. D., A. S. Brandstaetter, and C. J. Kleineidam. 2007. Reformation process of the neuronal template for nestmaterecognition cues in the carpenter ant Camponotus floridanus. Iournal of Comparative Physiology A 193:993-1000.

Liebert, A. E., and P. T. Starks. 2004. The action component of recognition systems: a focus on the response. Annales Zoologici Fennici 41:747-764.

Löfqvist, J. 1976. Formic acid and saturated hydrocarbons as alarm pheromones for the ant Formica rufa. Lournal of Insect Physiology 22:1331-1346.

Mizunami, M., N. Yamagata, and H. Nishino. 2010. Alarm pheromone processing in the ant brain: an evolutionary perspective. Frontiers in Behavioral Neuroscience 4:28.

O'Rourke, F. J. 1950. Formic acid production among the Formicidae. Annals of the Entomological Society of America 43:437-443.

Pophof, B. 2000. Octopamine modulates the sensitivity of silkmoth pheromone receptor neurons. Journal of Comparative Physiology A $186: 307-313$.

- 2002. Octopamine enhances moth olfactory responses to pheromones, but not those to general odorants. Journal of Comparative Physiology A 188:659-662.

R Development Core Team. 2017. R: a language and environment for statistical computing. R Foundation for Statistical Computing, Vienna.

Reeve, H. K. 1989. The evolution of conspecific acceptance thresholds. American Naturalist 133:407-435.

Rossi, N., D. Baracchi, M. Giurfa, and P. d'Ettorre. 2018. Data from: Pheromone-induced accuracy of nestmate recognition in carpenter ants: simultaneous decrease of type I and type II errors. American Naturalist, Dryad Digital Repository, https://doi.org/10.5061 /dryad.14k55m8.
Rossi, N., P. d'Ettorre, and M. Giurfa. 2018. Pheromones modulate responsiveness to a noxious stimulus in honey bees. Iournal of Experimental Biology 221:ieb.172270.

Sachse, S., and C. G. Galizia. 2002. Role of inhibition for temporal and spatial odor representation in olfactory output neurons: a calcium imaging study. Journal of Neurophysiology 87:1106-1117.

Seigneuric, A., K. Durand, T. Jiang, J.-Y. Baudouin, and B. Schaal. 2010. The nose tells it to the eyes: crossmodal associations between olfaction and vision. Perception 39:1541-1554.

Sherman, P. W., H. K. Reeve, and D. W. Pfennig. 1997. Recognition systems. Pages 69-96 in J. Krebs and N. Davies, eds. Behavioural ecology: an evolutionary approach. Blackwell Science, Oxford.

Starks, P. T., D. J. Fischer, R. E. Watsin, G. L. Melikian, and S. D. Nath. 1998. Context-dependent nestmate discrimination in the paper wasp, Polistes dominulus: a critical test of the optimal acceptance threshold model. Animal Behaviour 56:449-458.

Starks, P. T., R. E. Watson, M. J. Dipaola, and C. P. Dipaola. 2010. The effect of queen number on nestmate discrimination in the facultatively polygynous ant Pseudomyrmex pallidus (Hymenoptera: Formicidae). Ethology 104:573-584.

Stroeymeyt, N., F. J. Guerrieri, J. S. van Zweden, and P. d'Ettorre. 2010. Rapid decision-making with side-specific perceptual discrimination in ants. PLoS ONE 5:e12377.

Stumper, R. 1952. Quantitative data on the secretion of formic acid by ants. Comptes Rendus Hebdomadaires des Seances de l'Academie des Sciences 234:149-152.

Tedjakumala, S. R., M. Aimable, and M. Giurfa. 2014. Pharmacological modulation of aversive responsiveness in honey bees. Frontiers in Behavioral Neuroscience 7:221.

Tibbetts, E. A. 2002. Visual signals of individual identity in the wasp Polistes fuscatus. Proceedings of the Roval Society B 269:14231428.

Urlacher, E., B. Francés, M. Giurfa, and J. M. Devaud. 2010. An alarm pheromone modulates appetitive olfactory learning in the honeybee (Apis mellifera). Frontiers in Behavioral Neuroscience 4:1-10.

vander Meer, R. K., D. Saliwanchik, and B. Lavine. 1989. Temporal changes in colony cuticular hydrocarbon patterns of Solenopsis invicta. Journal of Chemical Ecology 15:2115-2125.

Van Swinderen, B., and R. Andretic. 2011. Dopamine in Drosophila: setting arousal thresholds in a miniature brain. Proceedings of the Roval Societv B 278:906-913.

van Zweden, J. S., and P. d'Ettorre. 2010. Nestmate recognition in social insects and the role of hydrocarbons. Pages 222-243 in G. J. Blomquist and A.-G. Bagnères, eds. Insect hydrocarbons: biology, biochemistry, and chemical ecology. Cambridge University Press, Cambridge.

van Zweden, J. S., S. Dreier, and P. d'Ettorre. 2009. Disentangling environmental and heritable nestmate recognition cues in a carpenter ant. Journal of Insect Physiology 55:159-164.

Vergoz, V., H. A. Schreurs, and A. R. Mercer. 2007. Queen pheromone blocks aversive learning in young worker bees. Science 317:384386.

Wilson, E. O. 1971. The insect societies. Harvard University Press, Cambridge.

Wyatt, T. D. 2014. Pheromones and animal behavior: chemical signals and signatures. 2nd ed. Cambridge University Press, New York.

Associate Editor: Madeleine Beekman Editor: Daniel I. Bolnick 